\title{
Vesico-uterine Fistula Following C-section - A Case Report and Literature Review
}

\author{
ROXANA CARAMAN ${ }^{1}$, ADRIAN TOMA ${ }^{1}$, IRINA BALESCU ${ }^{2}$, OVIDIU STIRU $^{3,4}$, \\ CORNEL SAVU ${ }^{5,6}$, CAMELIA DIACONU $^{7,8}$ and NICOLAE BACALBASA ${ }^{1,9,10}$ \\ ${ }^{1}$ Department of Obstetrics and Gynecology, “Dr. I. Cantacuzino” Clinical Hospital, Bucharest, Romania; \\ ${ }^{2}$ Department of Surgery "Ponderas” Academic Hospital, Bucharest, Romania; \\ ${ }^{3}$ Emergency Institute for Cardiovascular Diseases "Prof. Dr. C.C. Iliescu”, Bucharest, Romania; \\ ${ }^{4}$ Department of Cardio-Thoracic Pathology, "Carol Davila” University of Medicine and Pharmacy, Bucharest, Romania; \\ ${ }^{5}$ Department of Thoracic Surgery, "Marius Nasta” National Institute of Pneumophtisiology, Bucharest, Romania; \\ ${ }^{6}$ Department of Thoracic Surgery, "Carol Davila” University of Medicine and Pharmacy, Bucharest, Romania; \\ ${ }^{7}$ Department of Internal Medicine, "Carol Davila” University of Medicine and Pharmacy, Bucharest, Romania; \\ ${ }^{8}$ Department of Internal Medicine, Clinical Emergency Hospital of Bucharest, Bucharest, Romania; \\ ${ }^{9}$ Department of Visceral Surgery, Center of Excellence \\ in Translational Medicine "Fundeni" Clinical Institute, Bucharest, Romania; \\ ${ }^{10}$ Department of Obstetrics and Gynecology, "Carol Davila" \\ University of Medicine and Pharmacy, Bucharest, Romania
}

\begin{abstract}
Background/Aim: Vesico-uterine fistulas represent a rare type of genito-urinary fistulas; however, due to the increasing incidence of Caesarean section ( $C$-section) in the last decade, this abnormal communication between the urinary and genital tracts has been reported more often after such surgical procedures. The aim of the current article was to report the case of a 28-year-old patient who was submitted to surgery for a vesico-uterine fistula seven years after a C-section. Case Report: The 28-year-old patient with a previous history of four vaginal deliveries and one $C$-section was self-presented to the Gynecology Department for cyclic hematuria and diagnosed with a vesico-uterine fistula after injecting methylene blue in the uterine cavity during hysteroscopy. The patient was further submitted to surgery, and a parcelar myometrectomy en bloc with parcelar cystectomy, cystography, and prophylactic salpingectomy was performed. The postoperative outcome was uneventful. Conclusion: Although vesico-uterine fistulas represent rare events, they should be considered, especially in young patients with a previous history of $C$-section.
\end{abstract}

This article is freely accessible online.

Correspondence to: Irina Balescu, "Ponderas" Academic Hospital, Nicolae Caramfil street, 120143, Bucharest, Romania. Tel: +40 724077709, e-mail: irina.balescu@ ponderas-ah.ro

Key Words: C-section, vesico-uterine fistula, cyclic hematuria, parcelar myometrectomy, parcelar cystectomy.
Abnormal communications between the urinary and gynecological tracts are usually encountered in cases presenting locally advanced uro-gynecological malignancies such as cervical cancer (1). In addition, such abnormal communications are encountered after previous irradiation for pelvic malignancies and are most often located between the urinary bladder and vagina $(2,3)$. However, in other population subgroups, the most frequently encountered fistulas are vesico-uterine fistulas, especially in younger women with previous history of pelvic surgical procedures (4-7). Therefore, it is estimated that less than $5 \%$ of all urogenital fistulas are represented by vesico-uterine fistulas; however, due to the increasing incidence of surgical procedures at the level of the pelvic area among young patients, especially due to the increasing number of $\mathrm{C}$ sections, the number of vesico-uterine fistulas has reported an ascendant trend. In this respect, it should be noted that up to $88 \%$ of all these abnormal communications are related to C-sections $(7,8)$. The aim of the current paper was to report the case of a 28-year-old patient with a previous history of four vaginal births and one $\mathrm{C}$-section, who was diagnosed with a vesico-uterine fistula seven years after the C-section.

\section{Case Report}

The 28-year-old patient with previous history of four spontaneous vaginal deliveries and one $\mathrm{C}$-section seven years previously was self-presented for pelvic pain, menouria, and cyclic hematuria. The paraclinical tests revealed the presence 


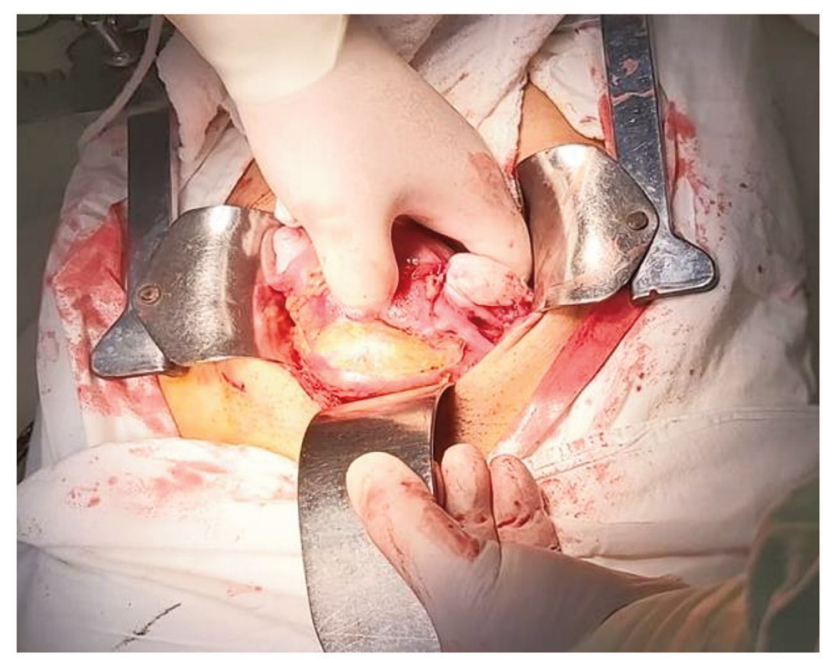

Figure 1. Intraoperative aspect. Strong adhesions between the urinary bladder and the uterine body are observed.

of anemia, with a hemoglobin level of $10.2 \mathrm{~g} / \mathrm{dl}$ in association with hematuria. In addition, the biochemical and microbiological analyses of urine revealed the presence of frequent leucocytes and nitrates in association with urinary infection by Escherichia Coli. The patient was further submitted to a transvaginal ultrasound, which revealed the presence of a thin, regular endometrial lining in association with diffusely irregular myometrium and a thickened urinary bladder wall. The patient was further submitted to a pelvic magnetic resonance imaging, which revealed the presence of thickened urinary bladder wall in close contact and with no demarcation line with the anterior surface of the uterine body, raising the suspicion of an abnormal communication at this level. To investigate whether this communication exists, the patient was submitted to hysteroscopy, which failed to demonstrate the presence of a visible communication between the two organs. However, when injecting methylene blue in the uterine cavity, blue urine was observed at the level of the urinary tube catheter demonstrating the presence of a vesico-uterine fistula. A control cystoscopy was performed and demonstrated the presence of a millimetric orifice through which methylene blue solution was observed. Therefore, the patient was submitted to surgery, strong adhesions being observed intraoperatively between the urinary bladder and the uterine body (Figure 1). During the process of adhesiolysis, the fistulous trajectory between the urinary bladder and the uterine body was identified. Most probably this abnormal communication was caused by the presence of a textiloma consisting of the suture stiches, which had been placed when

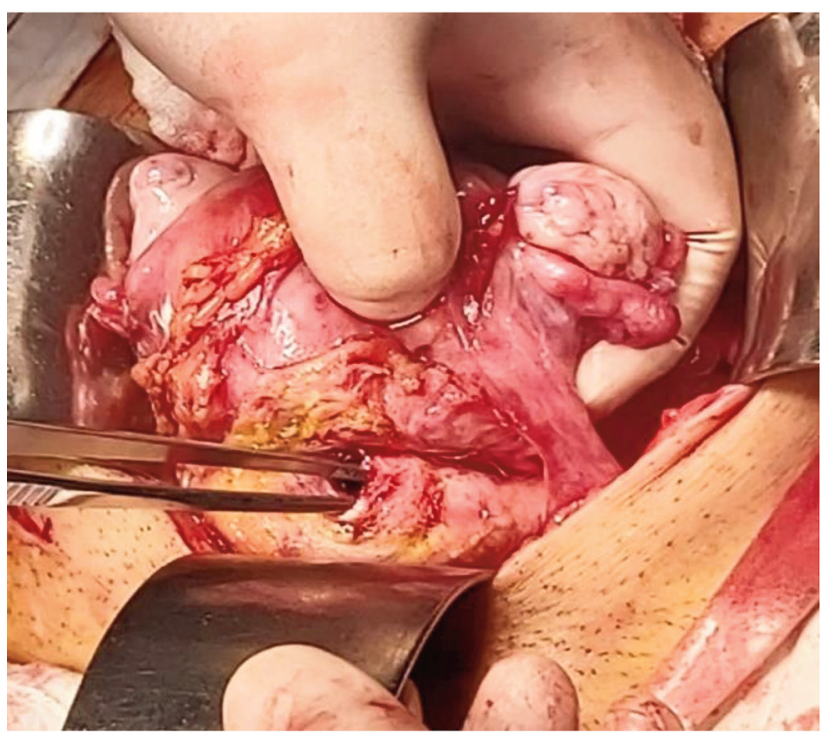

Figure 2. Identification of the fistulous trajectory between the uterine body and the urinary bladder.

suturing the uterine post $\mathrm{C}$-section incision (Figure 2 and Figure 3 ). The abnormal trajectory between the two organs was removed en bloc with partial cystectomy and cystography and partial myometrectomy with myometrial reconstruction while a flap of omentum was interposed between the uterine body and the urinary bladder (Figure 4 and Figure 5). In addition, due to the fact that the uterine cavity suffered significant modifications and the patient did not desire fertility preservation, prophylactic bilateral salpingectomy was performed. The patient had an uncomplicated postoperative evolution, being discharged with the urinary catheter in the fourth postoperative day. The histopathological report of the specimen excluded any sign of malignant transformation; the urinary catheter was removed at the $21^{\text {st }}$ postoperative day.

\section{Discussion}

The increasing incidence of $\mathrm{C}$-sections in the last decades resulted in a higher number of late postoperative complications such as vesico-uterine fistulas. In such cases, diagnosis is suggested by the patient's complains such as pelvic pain, amenorrhea, cyclic hematuria, menouria, secondary infertility or even spontaneous miscarriage (9). Association between amenorrhea and cyclic hematuria are known as Youssef syndrome and was initially described in 1957 (10). Depending on the competence of the uterine cervix, menouria and urinary incontinence might develop. Therefore, cases presenting an incompetent cervix present with exteriorization of urine through the vagina while cases 


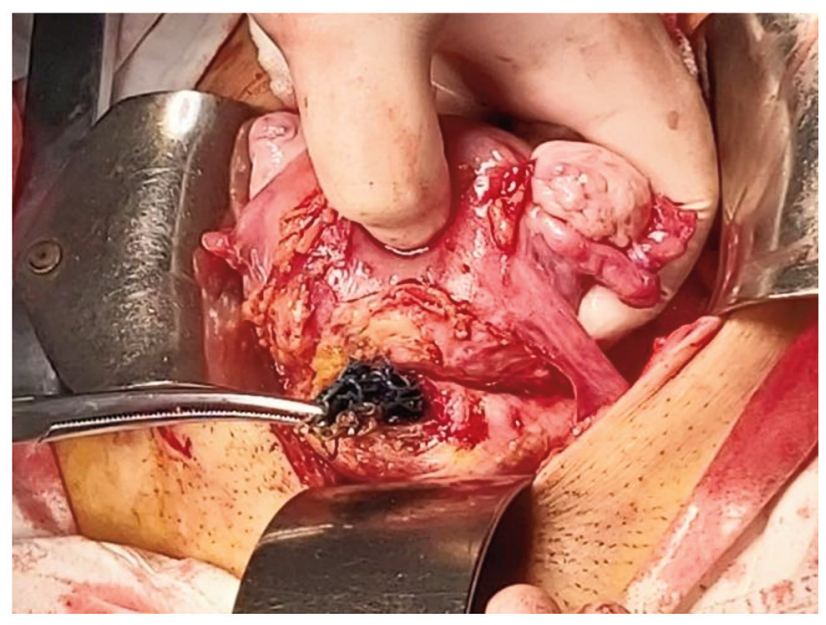

Figure 3. Extraction of the foreign body. The agglutination of stiches, which had been placed when suturing the uterine wall during the $C$ section is observed.

in which the cervix is competent will rather lead to the appearance of hematuria; in this respect three types of Youssef syndrome have been reported: type I syndrome associated with menouria, type II syndrome - associated with the apparition of urine loss at the level of the vagina and type III syndrome - defined by the presence of normal menstruation (11). In our case location of the leak in the supraisthmic area and association of a competent uterine cervix led to the appearance of hematuria with the absence of urinary incontinence (type I Youssef syndrome).

The risk factors that might lead to the development of such complications include the following: inadequate mobilization of the urinary bladder during $\mathrm{C}$-section, manual extraction of placenta, instrumental delivery, and pathological insertion of the placenta (9). However, uterinovesical fistulas can also occur after hysteroscopy, or in association with endometriosis, intrauterine device migration, inflammatory bowel disease or due to urinary bladder tuberculosis $(12,13)$.

The diagnostic methods of these pathological situations include intravenous urography, hysteroscopy, cystoscopy, transvaginal ultrasound or pelvic magnetic resonance imaging. While intravenous urography seems one of the most easily performed examinations, the rate of success is low due to the presence of a significant pressure gradient; at the level of the urinary tract a low level of pressure is encountered, at the level of the uterine corpus the pressure is significantly higher. Therefore, it is rather difficult to visualize a communication between the urinary bladder and the uterine body, although, theoretically, while a communication between the two organs exists, the contrast substance is expected to be found in both cavities.

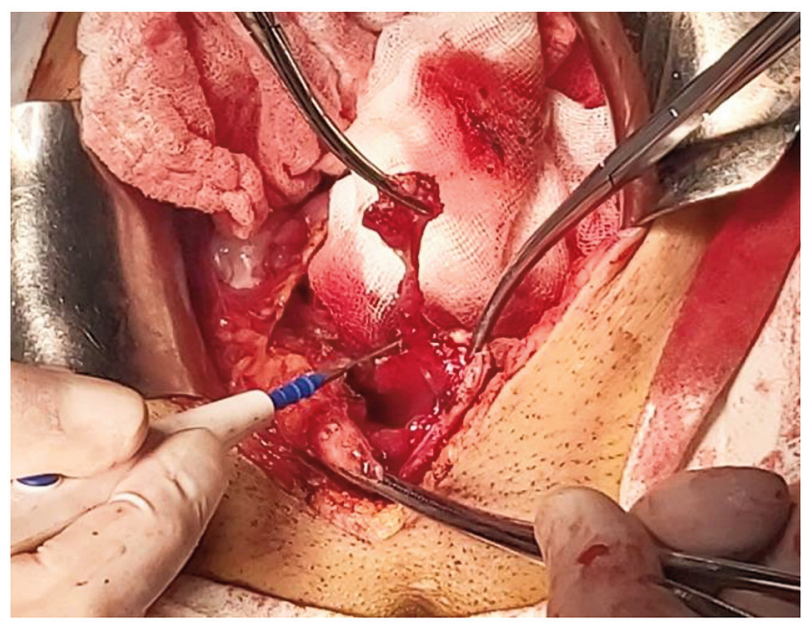

Figure 4. Removal of the fistulous trajectory by partial cystectomy with cystography.

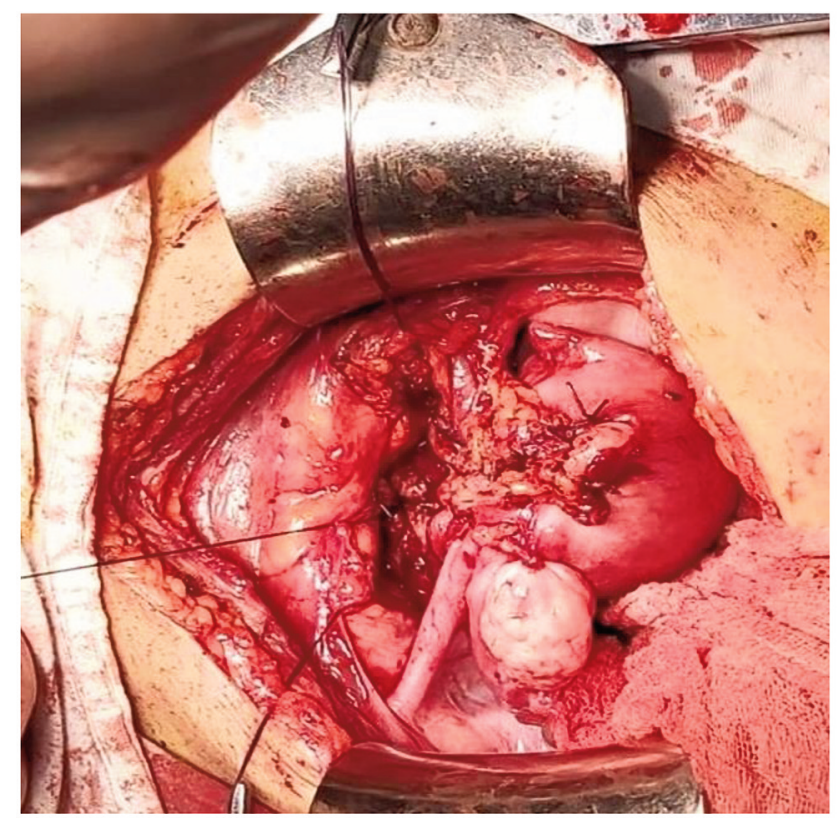

Figure 5. The final aspect after partial cystectomy with cystography, myometrectomy with myometrial reconstruction, and prophylactic bilateral salpingectomy.

Normally, the normal intravesical pressure is rarely elevated above the value of $20 \mathrm{~cm}$ of water during the fulfilling phase, while during the evacuation phase the detrusor muscle contraction will apply a maximal pressure of $50 \mathrm{~cm}$ of water; in the meantime, the uterine pressure varies between 35 and $160 \mathrm{~cm}$ of water according to the phase of the menstrual cycle. While during the proliferative 
phase intrauterine pressure ranges between 35 and $70 \mathrm{~cm}$ of water, during the secretory phase it ranges between 55 and $100 \mathrm{~cm}$ of water and during menstruation the intrauterine pressure reaches $130-160 \mathrm{~cm}$ of water (9). A similar problem is expected when performing a cystoscopy. However, in certain cases a pathological orifice might be discovered during cystoscopy, indicating an abnormal communication between the urinary bladder and the surrounding organs. Depending on the dimensions and location of this orifice, it can be dilated and visualized using the cystoscope in order to identify the length of the fistulous trajectory as well as the contralateral opening (1416). However, the most relevant imaging method to demonstrate the presence of utero-vesical fistulas remains pelvic magnetic resonance imaging, with an accuracy rate of up to $100 \%$ (14).

Regarding the time of development of such complications, short-term and long-term postoperative complications have been reported (5). The type of treatment can vary depending on the moment of the appearance of this complication. Therefore, in cases in which utero-vesical fistulas are diagnosed during the early postoperative period a conservative approach can be proposed (consisting of leaving in place the urinary catheter for a longer period of time). In cases in which this complication is encountered after a long period of time after surgery, conservative treatment is usually not efficient. In such cases, the abnormal communication usually occurs after significant local tissue modifications such as severe inflammation and fibrosis, which can no longer be treated in a conservative way; surgery consisting of fistulous trajectory removal en bloc with parcelar resection and reconstruction of the urinary bladder and of the uterine cavity is needed. According to the complexity of the case, surgery can be performed via open, laparoscopic, robotic approach, through cystoscopy or even through hysteroscopy $(17,18)$. When it comes to future fertility in cases in which this is desired, certain authors have also reported spontaneous pregnancy after such procedures (19).

As for the role of minimally invasive surgery, robotic approach has been recently shown to be associated with improved early- and long-term postoperative outcomes due to a better visualization of the structures and the presence of multiple freedom degrees, which are well recognized when using the robotic platforms $(20,21)$.

Another recent therapeutic strategy, which has been successfully implemented in patients with a long previous history of vesico-uterine fistulas, is represented by the transvaginal approach. Initially described in 2018 by Milani et al. (22), the method seems to be particularly effective due to the fact that it avoids the need to perform an extended intra-abdominal adhesiolysis, which could further increase the risk of other visceral injuries; therefore, by using a transvaginal approach, the pathway of dissection in order to reach the fistula might be shorter when compared to the standard abdominal approach. Moreover, certain authors also discuss its financial advantage when compared to robotic surgery as well as the cosmetic one, since during the transvaginal approach no abdominal incision is required (23). However, only few cases have been treated in this manner so far, the method being still under evaluation.

\section{Conclusion}

Vesico-uterine fistulas represent a rare type of genito-urinary complications. However, in the last decades, due to the increased number of $\mathrm{C}$-sections, an increasing trend has been reported. Depending on the time of diagnosis and severity of symptoms, a conservative treatment can be proposed. However, cases with a long evolution of the local inflammatory process have a lower chance of healing in a conservative manner. Therefore, in such cases radical surgical procedures such as fistulous trajectory removal en bloc with partial cystectomy and partial myometrectomy followed by local reconstruction of the urinary bladder and of the uterine cavity should be performed.

\section{Conflicts of Interest}

The Authors have no conflicts of interest to declare in relation to this study.

\section{Authors' Contributions}

$\mathrm{NB}$, AT performed the surgical procedure; IB, RC, OS, CS reviewed literature data; $\mathrm{CD}, \mathrm{RC}$ preoperative investigation of the patient; $\mathrm{CD}$, AT, NB postoperative follow up of the patient; IB, RC prepared a draft of the manuscript; NB reviewed the final version of the manuscript. All Authors read and approved the final version of the manuscript.

\section{References}

1 Moore KN, Gold MA, McMeekin DS and Zorn KK: Vesicovaginal fistula formation in patients with Stage IVA cervical carcinoma. Gynecol Oncol 106(3): 498-501, 2007. PMID: 17561235. DOI: 10.1016/j.ygyno.2007.04.030

2 Yen A, Tian Z, Hrycushko B and Albuquerque K: Risk factors for fistula formation after interstitial brachytherapy for locally advanced gynecological cancers involving vagina. J Contemp Brachytherapy 10(6): 510-515, 2018. PMID: 30662473. DOI: $10.5114 /$ jcb. 2018.80171

3 Emmert C and Köhler U: Management of genital fistulas in patients with cervical cancer. Arch Gynecol Obstet 259(1): 1924, 1996. PMID: 8933925. DOI: 10.1007/BF02505304

4 Ouedraogo B, Kirakoya B, Kabore M, Millogo A, Ouattara A and Kabore F: Vesicouterine fistula in Burkina Faso: Report of 36 cases in a multicentric study. Société Internationale d'Urologie Journal 2(4): 210-215, 2021. DOI: 10.48083/ NFMO2987 
5 Bağbancı MŞ, Emir ML, Dadalı $M$ and Karabulut A: Vesicouterine fistula, a rare cause of genitourinary fistula. Turk J Urol 40(4): 251-254, 2014. PMID: 26328188. DOI: 10.5152/ tud.2014.70846

6 Porcaro AB, Zicari M, Zecchini Antoniolli S, Pianon R, Monaco $\mathrm{C}$, Migliorini F, Longo $\mathrm{M}$ and Comunale L: Vesicouterine fistulas following cesarean section: report on a case, review and update of the literature. Int Urol Nephrol 34(3): 335-344, 2002. PMID: 12899224. DOI: 10.1023/a:1024443822378

7 Eogan $\mathrm{M}$ and McKenna P: Conservative management of a traumatic uterovesical fistula ('Youssef's syndrome'). Eur J Obstet Gynecol Reprod Biol 110(1): 114-116, 2003. PMID: 12932886. DOI: 10.1016/s0301-2115(03)00099-x

8 Machado Junior RA, Machado Junior LC and Lourenço LLE: Vesicouterine fistula (Youssef Syndrome): Case report and literature review. Rev Bras Ginecol Obstet 40(9): 563-569, 2018. PMID: 30231295. DOI: 10.1055/s-0038-1666998

9 Razzak L and Rizvi RM: Repair of vesicouterine fistula by not using traditional O' connor method. J Pak Med Assoc 71(1(A)): 143-145, 2021. PMID: 33484542. DOI: 10.47391/JPMA.019

10 Youssef AF: Menouria following lower segment cesarean section; a syndrome. Am J Obstet Gynecol 73(4): 759-767, 1957. PMID: 13411039 . DOI: $10.1016 / 0002-9378(57) 90384-8$

11 Petrikovets A and Lespinasse PF: Report of 2 cases of misdiagnosed vesicouterine fistula. Female Pelvic Med Reconstr Surg 20(6): e15-e17, 2014. PMID: 25185602. DOI: 10.1097/ SPV.0000000000000123

12 Keskin MZ, Budak S, Can E and İlbey YÖ: Incidentally diagnosed post-cesarean vesicouterine fistula (Youssef's syndrome). Can Urol Assoc J 9(11-12): E913-E915, 2015. PMID: 26834907. DOI: 10.5489/cuaj.3258

13 Birge O, Ozbey EG, Erkan MM, Arslan D and Kayar I: Youssef's syndrome following cesarean section. Case Rep Obstet Gynecol 2015: 605325, 2015. PMID: 26457214. DOI: $10.1155 / 2015 / 605325$

14 Abou-El-Ghar ME, El-Assmy AM, Refaie HF and El-Diasty TA: Radiological diagnosis of vesicouterine fistula: role of magnetic resonance imaging. J Magn Reson Imaging 36(2): 438-442, 2012. PMID: 22535687. DOI: 10.1002/jmri.23667
15 Fenkci IV, Demirbas M and Oztekin O: Sonohysterography in evaluation of Youssef's syndrome. Int Urogynecol J 21(5): 607608, 2010. PMID: 19894015. DOI: 10.1007/s00192-009-1029-0

16 Goel A, Goel S, Singh BP and Sankhwar SN: Cystographic images of Youssef syndrome: flower on top of the bladder. Urology 79(5): e69-e70, 2012. PMID: 22446343. DOI: 10.1016/ j.urology.2012.01.032

17 el-Lateef Moharram AA and el-Raouf MA: Retropubic repair of genitourinary fistula using a free supporting graft. BJU Int 93(4): 581-583, 2004. PMID: 15008734. DOI: 10.1111/j.1464-410x. 2003.04679.x

18 Abdel-Karim AM, Elmissiry M, Aboulfotoh A, Moussa A and Elsalmy S: Laparoendoscopic single-site surgery (LESS) and conventional laparoscopic extravesical repair of vesicouterine fistula: single-center experience. Int Urol Nephrol 45(4): 9951000, 2013. PMID: 23700107. DOI: 10.1007/s11255-013-0467-2

19 Rajamaheswari N and Chhikara AB: Vesicouterine fistulae: our experience of 17 cases and literature review. Int Urogynecol J 24(2): 275-279, 2013. PMID: 22592760. DOI: 10.1007/s00192012-1798-8

20 Baker MV, Kisby CK and Occhino JA: Vesicouterine fistula: A robotic approach. Int Urogynecol J, 2021. PMID: 34374804. DOI: $10.1007 / \mathrm{s} 00192-021-04940-6$

21 Govorov IE, Vorobeva MA and Komlichenko EV: Robotic repair of the symptomatic vesicouterine fistula. Int Urogynecol J 32(7): 1945-1947, 2021. PMID: 33078342. DOI: 10.1007/s00192-02004558-0

22 Milani R, Cola A, Frigerio M and Manodoro S: Repair of a vesicouterine fistula following cesarean section. Int Urogynecol J 29(2): 309-311, 2018. PMID: 29147755. DOI: 10.1007/ s00192-017-3506-1

23 Cao M, Zhang J, Chen Y and Liang Y: Transvaginal repair of vesicouterine fistulae: our experience of three cases. Int Urogynecol J, 2021. PMID: 34542661. DOI: 10.1007/s00192021-04973-x

Received September 5, 2021

Revised October 5, 2021

Accepted October 25, 2021 